la fàcheuse influence du poinçonnage des trous sans alésage consécutif.

Les trous de rivets de la virole en question avaicnt été simplement poinçonnés, et la figure 2 montre les amorces de criques près de ces trous. Au milieu de la virole existaient d'autres trous forés à la mèche.

Nous avons fait des essais de mandrinage sur un certain nombre de ces trous, en découpant autour des trous des rondelles de roo millimètres de diamètre, et en agrandissant lo trou central jusqu'à rupture de la rondelle. Le mandrin enployé pour agrandir les trous avait ses génératrices inclinées de $\mathrm{r} / 200^{\circ}$ sur l'axe, et on l'enfonçait très lentement à la presse.

L'une des rondelles, découpée près d'une cassure de la tỏlc, avait un trou poinçonné de $22 \mathrm{~mm}$. 5 de diamètre. Le mandrinage a fait casser net la rondelle presque immédiatemont, alors que le trou n'avait encore que $23 \mathrm{~mm} .2$ de diamc̀tre. Une autre rondelle, découpée au bord de la tôle, aulour d'un trou poinçonné de 20 millinètres do diamètre, s'est cassée net lorsque, par le mandrinage, le diamètre du tron est arrivé à 30 millimètres.

Par contre, des rondelles identiques, découpées autour de trous alésés de 22 millimètres de diamèlre, ne se sont rompues que lorsque les diamètres de ces lrous ont été agrandis à $55 \mathrm{~mm} .63$ et 69 millimètres, soit après un mandrinage de 150 à 200 pour $\mathbf{r} 00$ et plus.

Conclusions. - Nos conditions actuelles de réception des tòles auraient suffi pour nous permettre de rebuter immédiatement la tôle en question si elle nous avail été présentée. Cetle tôle fragile, si elle avait été employéc dans la construction d'unc chaudière à vapeur, aurait pu être cause de graves accidents. Pour les conduiles d'eau sous pression, les conséquences de l'emploi de tôles fragiles peuvent êlre ́galement désastreuses au point de vue des dégâts matéricls ct des chômages.

La róception des tòles en forge est donc absolument essenticlle pour éviter les accidents trop fréquomment constatés.

La constatation d'un écrouissage très nolable de la surface de la tôlc démontre, une fois de plus, la nécessité du recuit après laminage.

Fn outre, les essais de traction sur éprouveltes recuites ayant donné des résultats presque acceptables el même satisfaisants d'après certains cahiers des charges qui n'imposent que 20 pour roo d'allongement, on voit l'importance du mode de prélèvement des barreaux d'essai. Pour que les essais aient une réelle signification, et renseignent sur l'état do la tôle à réceptionner, il faut absolument que les éprouvelles soicnt prises sur la tôle après recuit, et non recuites á part, sans quoi on n'est pas sûr que le recuit de la tôle n'est pas négligé.

Il est à remarquer que les essais de choc sur barreaux entaillés, que nous demandons à litre de renseignement, donnent des différences relatives bien plus grandes que les essais de traction. Entre la résilience la meilleure, $\rho=5,49 \mathrm{du}$ barreau essayé tel quel, et la résilience $\rho=\mathrm{r}, 59 \mathrm{du}$ barreau recuit, nous avons une différence de 52 pour 100 , tandis que les allongements à la traction de 22,5 pour roo à l'état primitif et de 28 pour roo après remaniement no présentent que ig pour roo d'écart. Nous avons, du reste, constaté souvent que les résiliences en long et en travers d'une même tôle différaient beaucoup plus que les résistances et allongements à la traction en long et en travers.

Il semble donc que les essais de choc sur barreaux entaillés constituent un procédé de distinction relativement plus sen- sible que les essais de traction, bien que présentant avec cux une corrélation assez étroite, au moins en ce qui concerne le métal extra-doux qui nous intéresse.

Desjuzeur,

Directeur de l'Association lyonnaise des proprictaires dappareils à vapeur.

\section{DISTRIBUTIONS D'ÉNERGIE}

\section{ÉTAT ACTUEL DE L'INDUSTRIE DES CABI.ES ÉLECTRIQUES}

Communication faite à la Société Internationale des Electricuns par son président, M. Grosstilin

La question des défauts des canalisalions a loujours temu une grande place dans les soucis des exploitanls el, pour cux, les claquages de câbles ont été longlemps un alfolant cauchemar.

Celui qui, le premier, osa confier à un fil de cuivre entourśs d'une mince couche de papier l'énergie redoulable du courant à haule tension, aurail dù se mcltre au coeur une triple cuirasse d'acier, s'il avait pu prévoir tous les accidents qu'il allait provoquer. Par bonheur, les praticiens, inconscients des risques courus, allèrent de l'avanl sans demander l'avis préalable des théoriciens.

Dans la période héroïque, vers $\mathrm{r} 89^{2}$, alors qu'on commençail à distribuer du courant allernatif par câbles concentriques, presque chaque jour faisail éclore une série de défauts dispersés, sur toul te réseau, comme par le caprice de quelque démon malfaisant. Praticiens el théoriciens se rencontrèrent alors pour découvrir el sanclionner des prérautions qui se montrèrenl efficaces dans l'exploilalion des citbles concentriques.

On travaillait, dans ces temps reculés, aur lensions de 2000 à 3 ooo volts, qu'on qualifiail, avec un respeduemx effroi, d'énormes. Quand on s'enhardil assez pour alleindre 5 ooo volts, de nouveaux phénomènes apparurent : las câbles s'obstinaient à claquer quand on fermait un inter'upteur sur un réscau non chargé, on quand un courl circuil, mêne momentané, élail créé en un point quelconque. Souvenl, ce court circuit survenail en nême lemps dans mn alternalom, dans un câble el dans un lansformateur. Qui chail responsable, du consiructeur d'appareils ou du constructeur de chbles? L'exploilant, bon juge, partageail les écailles : il faisait payer l'un cl l'autre.

Ies constructeurs s'élonnaient, cependant, de voir lléchir en service des isolants qu'ils avaient essayés avec un coefficient de sécurité paraissant les mettre à l'abri de tout risque. Ils se tournèrent de nouveau vers les savants pour oblenir le mot de l'énigme el Je remède sauveur. La réponse ful donnée par les Polier, les Picou, les Blondel, les Boucherol, les Brylinski.

On reconnul que, le plus souvent, c'est l'exploilain qui était fautif en déchaînant des oscillations dans son réseau, comme M. Jourdain faisait de la prose, sans le savoir. Il fallur, dès lors, s'astreindre à surveilJer d'un peu près les conditions de régime, à rolever la forme des courbes de lension, à prendre de nombreuses et minulieuses précautions.

De leur côté, les constructeurs portèrent au triple le coeffcient d'essai des câbles qui n'était auparavant que du double. On put, sans danger, mettre à la ferraille le bric-à-brac on- 
combrant el dangereux des limiteurs, et les tensions d'emploi monlèrent graduellement, nous allons voir tout à l'heure jusqu'à qu'clle limile.

Toul en supprimant les limileurs, on dut conserver les parafoudres, pour protéger, contre les décharges atmosphériques, les canalisations souterraines directement reliées aux lignes aćriennes, car, si l'on supprime le parafoudre à ld liaison, la décharge passe à la terre par l'isolant du câble. Or. les parafoudres ne sont guère d'un fonctionnement plus sûr que les limiteurs : on en trouve la preuve dans la mulliplicilé des systèmes proposés.

De plus, lorsqu'i] s'agit de protéger une tráversée souterraine de ligne aérienne à plus de 20 ooo volts, on est conduit à installer à chaque exlrémité de véritables postes, dont le prix majore la dépense à faire d'aulanl plus lourdement que la traversće est plus courte. Mais, dans ce cas, du moins, il est une autrc solution du problème.

Les Américains onl observé que les lignes aćriennes isolées pour roo ooo volts n'onl pas à souffrir des orages, et ils se sont empressés de les débarrasser de leurs parafoudres. Si les propriétés de la foudre ne se modifient pas en passant l'Atlantique el si, d'aulre part, les fabricants de câbles peuvent livrer, à des prix acceplables, de courtes longueurs tenant soo ooo volls, le problème de l'établissement économique des traversées souterraines à l'épreuve de la foudre se trouvera résolu, du moins pour les lignes fonctionnant à moins de roo ooo volts, ce qui est encore le cas de presque toutes les lignes curopéennes $\left(^{1}\right)$.

Bicn entendu, lorsque la parlie souterraine constitue, non unc simple traversée de voie, mais un transport à distance nolable, il csl beaucoup plus économique de protéger pur un poste de parafoudres les câbles construits pour la tension jusle nécessairc. Nous sommes donc conduits à nous demander si les fabricants peuvent livrer des câbles à roo ooo volts.

Los câbles à conducteurs multiples construits pour foncliomner à Ioo ooo volts ne sont guère, jusqu'ici, sortis du laboraloire ou, s'ils l'ont fait, l'aventure n'a pas, en général, tourné à leur honneur.

L'an dernier, au Congrès de Turin, M. Iichtenstein, de la maison Siemens, annonçait que la tension de 40 ono volts élail le maximum industriellement possible pour les câbles à conducteurs multiples, mais que, pour les câbles à conducteur unique, on avait atteint en Allemagne 60 ooo volts. Il est facile, en effet, de se rendre compte que, pour une tension donnée, un câble est beaucoup plus facile à fabriquer quand il ne contient qu'un seul conducteur.

Tout d'abord, à diamètre extérieur égal, on peut donner à l'isolant une épaisseur beaucoup plus grande.. De plus, l'isolant au papier, qui est maintenant d'un emploi général, n'est imprégné qu'après avoir été enroulé à sec sur chaque conducleur. I'imprégnation est d'autant plus aisée à réussir que là masse tolale de papicr est moindre : or, celle-ci esl, à ćpaisscur d'isolant ćgale, trois fois plus petite pour un câble ì conducteur unique que pour un câble à trois conducteurs. Ceci explique la constatation faite par M. Lichtenstein. Mais, d'après ses dires, les transmissions à ron ooo volts seraicnt encore irréalisables.

Mon rapport, à ce même Congrès de Turin, annonçait que des maisons françaises avaient construit quelques kilomètres de câbles à trois conducteurs réunis sous une même enveloppe et fonctionnanl à 60 ooo volts, soit à une tension supé-

(1) Il n'a encore été signalé, en Europe, comme ligne aérienne à tro ooo volts, que celle de Lauchammer, en Allcmagne. rieure de 5o pour soo au maximum atteinl par les Allemands, pour les câbles à conducteurs multiples. Ne nous privons pas de signaler au passage celte avance de l'industrie francaise sur ses rivales, avance d'autant plus brillante que les autres pays viennent bien après l'Allemagne dans cet ordre d'idées.

Les Américains, beaucoup moins soucieux que nous d'csthétique, el même de sécurité, metlent sur poteaux presque trutes leurs lignes à haute tension : ils n'ont donc pas poussé la fabricalion des câbles au-delà des tensions de 200000 25 ooo volts. Il en est à peu près de même en Angleterre, oì les distances étant moins longues, les tensions employécs sont moins élevées.

Les Italiens n'ont pas dépassé 30 ooo volts.

Les maisons françaises faisant le càble à trois conducteurs pour 60 ooo volts, sont à même de livrer aussi le conducteur unique isolé pour too ooo volts. Les raisons sommairement indiquées tout à l'h.cure vous expliquent pourquoi. On peut donc compter sur nos fabricanls de câbles pour assurer l'établissement, sans parafoudres, de traversées ou même de transports jusqu'à roo ooo volts, à condition d'cmplojer des câbles à conducteur unique, sans armure de fer ; cette dernière, yous le savez, occasionnerait des pertes d'énergie considérable dans les transmissions de courant alternatif.

Pcut-on dépasser cette limite et prévoir la pose de traversées ou de lignes à plus de roo ooo volts? Nous avons tous été élevés dans l'idée que la crainte de l'extrapolation est le commencement de la sagesse, et je ne voudrais rien affirmer ru-delà des limites vérifiées par l'expérience. C'est donc de. slle-ci qu'il faut attendre la réponse.

Ayons, d'ailleurs, la franchise de reconnaître que les câbles armés employés maintenant sont des produits industriels de la dernière barbarie. Nous fabriquons, de toutes pièces, des diélectriques d'une homogénéité incertaine, si fragiles, si perméables, que nous devons, pour leur assurer unc durée indéfinie, les enfermer dans une boîte de conscrves et cette boîte n'est pas même en fer-blanc. Ellc est en plomb, et si ce plomb présente l'avantage précieux d'une étanchéité parfaite, il est en revanche coûteux, lourd, aussi mou que l'isolant qu'il protège, en sorte qu'il doit être revêtu lui-même d'une cuirasse d'acier, laquelle à son tour devra être mise à l'abri de l'oxydation. Nous venons de voir que cettc cuirasse doit être rejetée pour les câbles à conducleur unique transmettant des courants alternatifs. On la remplace par des tuyaux de grés, de ciment, de maçonnerie qui augmentent notablement le prix du câble posé.

Le prix de revient d'un transport souterrain à très haute tension est finalement de deux à trois fois supérieur à celui d'un transport aérien de même puissance, et l'on ne songerait pas à l'établir s'il ne supprimait complètement les frais d'entretien qui grèvent lc transport aérien. Mais l'inconvénient principal de l'enveloppe de plomb consiste dans la capacité dont elle dote le câble, capacité dont les effets, pour les transports de plus de roo $\mathrm{km}$., sont fort difficiles à prévoir. Il en est un, du moins, qui paraît inévitable, c'est le courant de charge.

Une ligne de 50 millimètres carrés, à la fréquence de 25 el à la tension de roo ooo volts, de $400 \mathrm{~km}$. de longueur, prendrait à vide 600 ampères, soit 60 ooo kilovolts-ampères. Peut-être pourra-t-on réduire cet énorme courant de charge par des dispositifs tels que des bobines de réactance ou en répartissant artificiellement le débit suivant les heures de la journée, de manière à ne jamais marcher à vide. 
Par malheur, la capacilé, qu'il faul évaluer, au minimum, i lo microfarads dans le cas que je riens de citer, n'est pas localisée comme celle d'un condensalem. Elle esl répartic lo long du conducteur, ct nous ne savons guère comment flle so comportera. Sans doule, M. Brylinski nous a dommé quel(que réconfort en nous montrant qu'unc capacité r'épartie amortit les harmoniques sur les longues lignes, mais il nous a dit aussi qu'elle détermine une infinité de périodes de résonance.

Qu'arrivera-t-il au passage d'une oscillation ? Ics surtensions seront-elles amorties ou amplifiées ? Les dipositifs compensaleurs du courant de charge resteront-ils cfficaces? Arouons que les exploilanls ont quelque excuse d'hésiter à risquer un capital important avant d'avoir ćté rassurés sur lous ces points.

La solution la plus sûre du problème serail la découverle d'un isolanl souple, imperméable, qui libérerait les câbles ic leur cuirasse el de leur capacité. (Je ne parle pas du cantchouc Para, dont le prix est tel que les fabricants de câbles onl depuis longtemps renoncé même à on voir). Jo suis persuadé que, tôt ou tard, paraîtra cet oiseau blcu des isolants.

Pendant des milliers d'années, nos ancêtres ont en vain rếé à la conquête de l'air et le jour où, cessant de rêver, quel(pues chercheurs ont voulu, pour tout de bon, réussir, on a vu se lever de la vieille terre l'admirable vol des aćroplanes.

Vous me trouvez bien prétenlicux d'évoquer les avions à propos de câbles. Cependant, pour avoir moins de panache fl do cocarde, l'enjeu poursuivi par les câbleur's n'est guèrc noins cxcitant.

Paris manquera quelque jour d'énergic électrique, si l'on nc va pas en prendre pour lui à la houille blanche les Alpes, ou à la houille noire du Pas-de-Calais, et de grands projets sont, depuis des années, à l'élude.

Croyez-vous prudent, possible mème, de confier à un fil do cuive tendu sur des cloches fragiles que portent des pylones ballus par les vents, exposés à la foudre, aux balles des ennemis du dehors et du dedans, l'alimentation de Paris er énergie ?

Récemment, le Sud-Lumière se décidail, à la suite de sabolages audacieux, à enterrer ses feeders qui vont jusqu’à Corbcil. Son exemple scra suivi, tôt ou tard, pour les longs liansports.

Nos procédés actuels ne nous permettenl pas d'aborder les lensions supéricures à xoo ooo volls en dehors du système continu série, parfait pour les câbles, mais bien dangereux pour les machines. Nous trouverons done des procódés nouveaur ou rous perfectionnerons nos mélhodes artuclles, car c’út une nécessité absoluc.

Fn atlendant, nous vous demanderons d'étudier de ylus près encore, par le calcul et par l'expérience, les phénomònes facheux qui nous guettent, afin de prévoir toules les prórautions à prendre si nous devons aborder Jes longs transports avec nos procédés d'aujourd'hui. Fl n'est-ce pas là un admirable sujet à soumettre a volre Section des canolisations ?

\section{Congrès national pour la défense et le développement du Commerce extérieur.}

A lieu à Paris, du 24 au 27 juin, au Conservatoire des Arts et Métiers. - A l'ordre du jour: Etude des voies de transport (Chemins de fer et navigation) entre Marseille, Lyon et Genève, et entre Bordeaux, Lyon et Genève.

\section{ACADÉMIE DES SCIENGES}

MÉCANIQUE ET ÉLECTAICITÉ

Résolution graphique de l'équation trinome à exposants quelconques. Note de M. Rodolphe Soncac. Sćance du 25 mars 19 I2.

L'équation trinome pent toujours se ramener à la forme :

$$
\pm a z^{\gamma} \pm b z^{\xi}= \pm 10^{p}
$$

$a, b, x, \beta$ étant des nombres posilifs.

Posons :

$$
a z^{\alpha}=\Lambda \quad b z^{5}=B
$$

d’ồ :

$$
\pm A \pm B= \pm 10 p
$$

$$
a z^{\alpha}=A \text { donne } \log a+\alpha \log z=\log A
$$

que l'on pentécrire: $\quad(x) \quad(y) \quad(t)$

$$
\left|\begin{array}{rrc}
0 & -\log z & 1 \\
1 & \log A & 1 \\
1 & \log a & 1+\varkappa
\end{array}\right|=0
$$

Ce déterminant exprimo l'alignement des pointa :
(z) $\left\{\begin{array}{l}x=0 \\ y=-\log z\end{array}\right.$
(1) $\left\{\begin{array}{l}x=1 \\ y=\log 1\end{array}\right.$
(P) $\left\{\begin{array}{l}x=\frac{1}{1+a} \\ y=x \log a\end{array}\right.$

Il suffit de construire les échelles $: y=-\log z, y=\log A$, el le réscau de paralliles : $x=\frac{1}{1+x}$

Le point figuratif $P$ des domnées $a, \alpha$ est à Tinlersection de la droile eotée s arec la radiante qui joint lorigine () des coordoncés cotée $z=1$ ) au point de cole $1=a$.

On a de même le point ( figuratif des données $b, \beta$.

Les points $P$ et $Q$ élant ainsi déterminés, deux droiles tournant. antour de ces points, el sc coupant sur l'échelle (z), donnent une solution de l'éfuation trinome quand les rotes du segment qu'elles interceptent sur l'échelle (.1) satisfont à la relation ( 1 ).

Si l'on doit faire un fréquent usage de l'abaque, il sera commode de construire, en celluloüd ou en mica, un compas dont l'arliculation se termine en poinle; les branches, qu'um caoulchone tend à rapprocher, s'appuicront contre deux fines aiguilles piquécs aux points $P$ et $Q$. Il suffira de déplacer la pointe sur $(z)$ jusqu'à ce que les branches interceptent sur $(A)$ Je segment voulu.

On rappelle que $M$. Torrès a donné un autre procédé de résolution, à l'ajle de trois échelles logarithmiques, moliles lo long de leur support et dans le sens perpendiculaire. Ces savant a conçu une intrénieuse machine qui permel d'effetuer mécaniquement les opérations.

Généralisation de la construction de Massau, et abaque pour résoudre les équations de la forme:

$$
z^{\alpha+\beta}+n z^{2+\beta}+p z^{z^{\beta}}+q=0
$$

Note de U. R. Sorear. (Voir les Comples Remders de l'Aendémir des Sciences, r 1 mars $\mathrm{r} 9 \mathrm{r}$, page 686$)$.

Distribution des déformations dans les métaux soumis à des efforts. Cas du plissement des tuyaux. Note de Ch. Frémont. Séance du 2 ? avril ig 92.

Si l'on prend un cylindre creux J'un mélal plastique homogène, et d'une épaisseur uniforme, un tronçon de tuyau limité par deux sertions droites, et quon le eomprime perpendiculairement à son axe entre les plateaux d'une presse, on sait qu'il se forme à l'une des extrémités, sans qu'il soit nécessaire de guider le métal par un mandrin, ou par tout autre procédé, un premier bourrelet auquel, successivement, ef un à un, viennent s'en ajouter d'autres, jusqu'ì ce que le tube soil plissé sur tonte sa longueur ( ${ }^{1}$ ).

(1) Engineering du to Janvier igo8, page 38. 